\title{
Aorto-esophageal fistula associated with vascular ring: Double the arch trouble
}

\author{
Umar S. Boston ${ }^{* 1,2}$, Peter E. Fischer ${ }^{2}$, Shyam K. Sathanandam², Christopher J. Knott-Craig ${ }^{1,2}$ \\ ${ }^{1}$ LeBonheur Children's Hospital, United States \\ ${ }^{2}$ University of Tennessee Health Science Center, United States
}

Received: July 9, 2017

DOI: $10.5430 /$ css.v3n3p24
Accepted: August 16, $2017 \quad$ Online Published: August 26, 2017

URL: https://doi.org/10.5430/css.v3n3p24

\begin{abstract}
Aorto-esophageal fistula (AEF) is a rare form of upper gastrointestinal bleeding that is associated with high mortality if not surgically addressed. It is most commonly associated with descending thoracic aneurysms and foreign body ingestion. We report a case of surgical treatment of AEF associated with double aortic arch vascular ring.
\end{abstract}

Key Words: Vascular ring, Double aortic arch, Esophageal fistula, Hemoptysis

\section{INTRODUCTION}

Aorto-esophageal fistula (AEF) is a life threatening condition associated with high mortality, and is most commonly associated with descending thoracic aortic aneurysm or ingestion of foreign body. ${ }^{[1,2]}$ Here in we present a case report of AEF in association with a double aortic arch vascular ring.

\section{CASE REPORT}

A 36-year-old male sustained multiple gunshot wounds to the chest and abdomen. He initially underwent abdominal exploration with uretostomy and colostomy placement, followed by median sternotomy and repair of a bullet injury to the right ventricle. Tracheostomy for respiratory insufficiency was required due to acute respiratory distress syndrome.

In the post-operative period he developed massive hemoptysis requiring 80 units of blood. This was initially thought to be related to a tongue laceration, for which he had emobolization of his lingual arteries. A chest computed tomography (CT) scan was obtained due to recurrent massive hemopty- sis. This revealed a vascular ring in the form of a double aortic arch (see Figure 1) and a fistula between the distal left aortic arch and the esophagus (see Figure 2). A SengstakenBlakemore tube was placed for ongoing massive hemoptysis allowing for resuscitation and urgent timing of operation.

Percutaneous stenting was not an option due to the concerns for obstruction of the right arch as well as the high risk of infection when placing a stent into an infected field.

The patient was taken to the operating room urgently for repair of AEF. He underwent left thoracotomy via the fourth intercostal space. The mediastinum was hostile with a large phlegmon and lymph nodes around the aorta and esophagus. To better visualize the fistula the left/anterior aortic arch was dissected and transected distal to the left subclavian artery. The AEF was then addressed. To prevent exsanguination proximal control was obtained by dissecting out the right posterior arch and distal control was obtained by dissecting out the descending thoracic aorta. The aorta was clamped in these 2 areas for a period of 26 minutes. The fistula was then

\footnotetext{
*Correspondence: Umar S. Boston; Email: uboston@uthsc.edu; Address: LeBonheur Children's Hospital/University of Tennessee Health Science Center, United States.
} 
safely dissected out, separating esophagus from aorta. The $6 \mathrm{~mm}$ aortic defect was repaired primarily. The linear tear in the esophagus was $2.5 \mathrm{~cm}$ and was repaired primarily over a Montgomery T-tube which was externalized through the $7^{\text {th }}$ intercostal space.

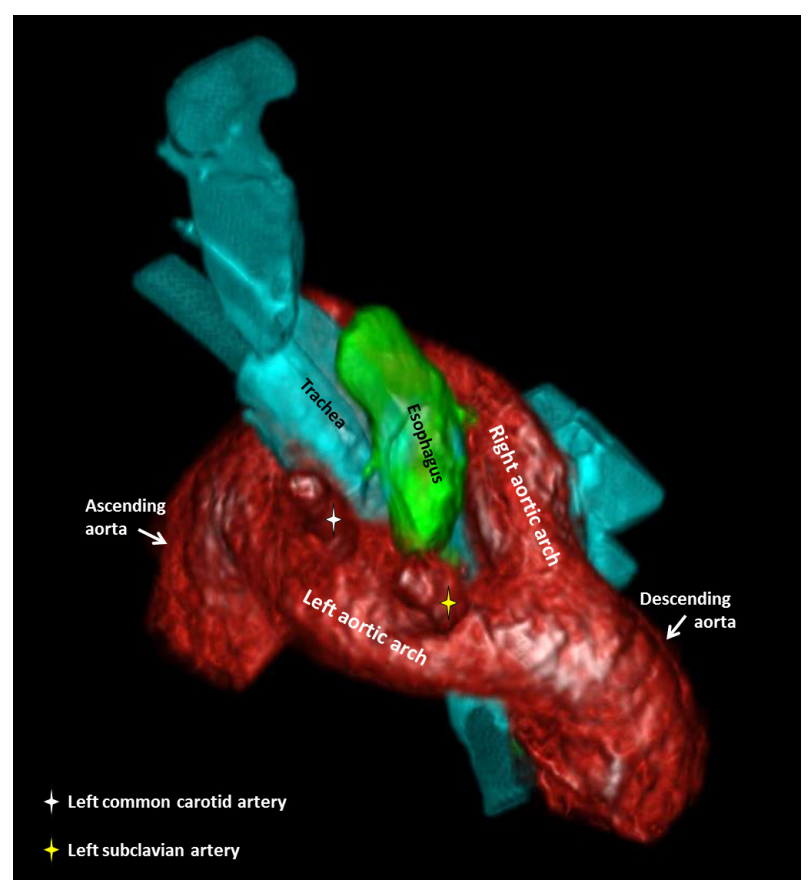

Figure 1. A steep LAO-cranial projection of a 3D-reconstructed image from an axial CT-scan of the chest demonstrating the double aortic arch forming a vascular ring around the trachea and the esophagus

An intercostal muscle flap was then developed on its vascular pedicle and used as an interposition autologous flap between aorta and esophagus to prevent recurrence of the fistula. Chest tubes were placed and the thoracotomy was closed.

The patient's recovery was protracted due to his multiple gunshot wound injuries compounded by his AEF. He ultimately succumbed to his multiple injuries 5 weeks following AEF repair.

\section{Discussion}

This is the first case report of a double aortic arch with concomitant AEF. The etiology of the AEF can be surmised by the following: the patient had a long-standing history of dysphagia related to his vascular ring. CT scan confirmed vascular ring compression of the esophagus. The inciting event for the AEF formation is believed to be related to an esophageal tear due to traumatic nasogastric tube insertion which eventually resulted in the fistulous communication with the aortic arch.

Published by Sciedu Press

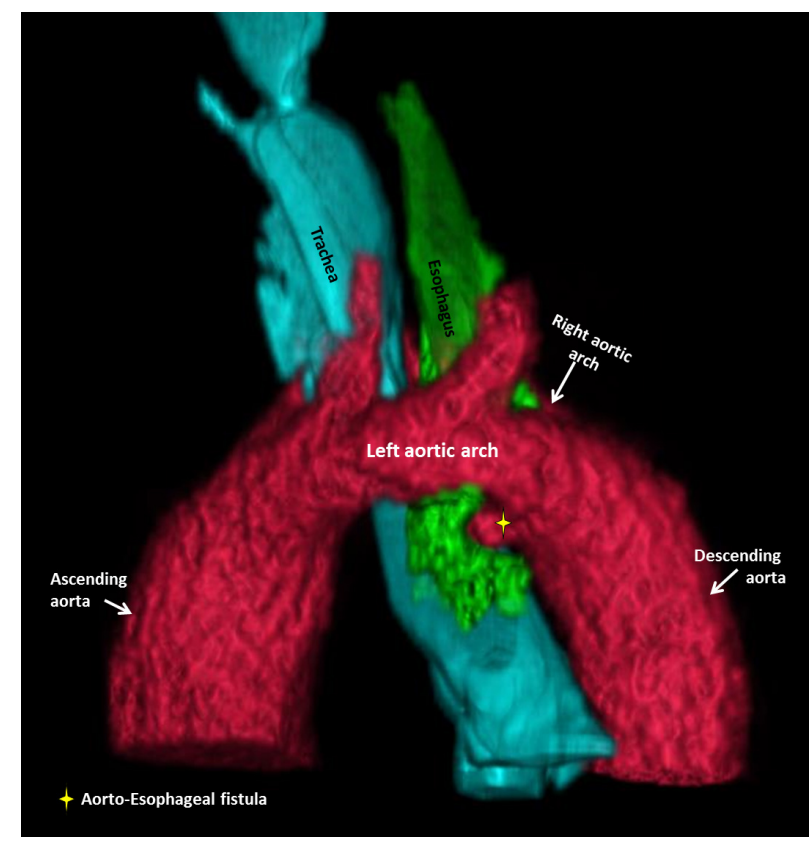

Figure 2. A $90^{\circ} \mathrm{LAO}$ projection of a 3D-reconstructed image from an axial CT-scan of the chest demonstrates the aorto-esophageal fistula from the junction of the left aortic arch and descending thoracic aorta to the esophagus

Chiari in 1914 was the first to report AEF. ${ }^{[3]}$ To date there has been over 500 cases of AEF reported in the literature with a very high associated mortality. ${ }^{[4]}$ Despite the etiology of the AEF there are a number of critical decisions that need to be considered.

This patient's demise resulted from multiorgan failure which potentially could have been averted with an earlier presentation. However there were a number of important teaching points from a surgical perspective in addressing this very challenging case: (1) avoidance of cardiopulmonary bypass in a patient who had sustained severe gunshot wound injuries and had required massive resuscitation for episodes of exsanguination leading to lung and renal dysfunction and massive volume overload; (2) good exposure of the AEF was critical and necessitated division of the left anterior arch. A maneuver that is performed for double aortic arch vascular ring repair; (3) proximal and distal control of aorta was essential to avoid exsanguination upon fistula take down. This necessitated dissection of the right arch and proximal descending aorta; (4) An esophageal diversion strategy to allow for healing of the esophagus is also critical. In this case a T-tube was used, however primary repair with proximal diversion using a neck spit fistula is another reasonable approach; (5) Finally, an interposition pedicled muscle flap was placed to prevent AEF recurrence. We believe these principles are essential for the successful repair of AEF in the presence of a double aortic arch. 


\section{CONFlicts OF Interest Disclosure}

The authors declare they have no conflict of interest.

\section{REFERENCES}

[1] Reardon MJ, Brewer RJ, LeMaire SA, et al. Surgical Management of Primary Aortoesophageal Fistula Secondary to Thoracic Aneursym. Ann Thorac Surg. 2000; 69: 967-70. https://doi.org/10.101 6/S0003-4975 (99) 01087-5

[2] Toumi O, Jabra SB, Mahmoudi A, et al. Aorto-Esophageal Fistula: A Rare Fatal Case of Gastrointestinal Hemorrhage Caused by Foreign
Body. J of Surg. 2015; 3: 1-3. https://doi.org/10.11648/j.j s. 20150301.11

[3] Chiari H. Uber Fremdko rperverletzung des Oesophagus mit Aorten perforation. Berlin Klin Wschr. 1914; 51: 7-9.

[4] Hollander JE, Quick G. Aortoesophageal Fistula: A comprehensive review of the literature. Am J Med. 1991; 91: 279-287. https: //doi.org/10.1016/0002-9343(91)90129-L 